\title{
Incommensurately modulated structure - a means for increased luminescence efficiency in the solid state
}

\author{
A. Makal \\ Faculty of Chemistry, University of Warsaw, ul. Zwirki i Wigury 101, 02-089 Warsaw, Poland \\ amakal@chem.uw.edu.pl
}

Among inorganic and metal-organic materials, there are numerous instances where modulated structure determines material's unique and useful properties: (super)conductivity of layered cuprates[1], ferroelectricity of certain perowskites[2] or, in particular, quantum yields of fluorescence in complex molybdates and tungstates with the scheelite-type $\left(\mathrm{A}^{\prime}, \mathrm{A}^{\prime \prime}\right)_{\mathrm{n}}[(\mathrm{Mo} / \mathrm{W}) \mathrm{O} 4]_{\mathrm{m}}$ structures, where $\mathrm{A}^{\prime}, \mathrm{A}^{\prime \prime}=$ alkali, alkaline-earth or rare-earth elements[3]. One of the earliest and most important examples of modulations that relate to specific physical properties for organic compounds is the case of TTF-TCNQ cocrystals. Modulation of the crystal structure (i.e. a slip of the TTF molecules) at temperatures below $60 \mathrm{~K}$ results superconductivity of the system[4,5]. However, the general number of reported cases of organic modulated structures remains relatively low[6]. This is because interpretation of modulations in molecular crystals is very challenging: modulation affects positions and atomic displacement parameters of many atoms, while satellite reflections, necessary to describe them, are inherently less intense than the main reflections and therefore more difficult to collect or even detect during standard diffraction experiment. For instance, in the case of TTF-TCNQ, the strongest satellite reflections were 10000 weaker than Bragg reflections and required specific data reduction procedure[5].

This communication presents an instance where a pyrene-based fluorophore, 1-acetylpyrene (1AP), crystallized as incommensurately modulated polymorph which displayed particularly efficient luminescence in the solid state. Its crystal structure has been formerly solved and presented in its supercell approximation with $Z^{\prime}=6$. Since the compound has been shown to yield several polymorphs[7], the increased efficiency of luminescence in the modulated form can be attributed directly to the modulation in its crystal structure. Structural parameters most affected by the modulation are an interplanar distance in a 1AP dimer and relative lateral shift of its constituents. This results in the presence of an assembly of dimers in the crystal, varying slightly in the extent of orbital overlaps, which apparently broadens the range of effective UV absorption in the sample.

[1] (a) Y. Gao, P. Lee, P. Coppens, M. A. Subramania, A. W. Sleight, Science, 1988, 241, 954; (b) Y. Gao, P. Lee, J. Ye, P. Bush, V. Petricek, P. Coppens, Phys. C. Supercond., 1989, 160, 431; (c) A. V. Mironov, P. Coppens, N. R. Khasanova, E. V. Antipov, V. Petricek J. Solid State Chem., 1994, 109, 74.

[2] (a) J. P. Pouget, Phys. Rev. B, 2000, 62, 176; (b) D. A. Rusakov, A. M. Abakumov, K. Yamaura, A. A. Belik, G. Van Tendeloo, E. TakayamaMuromachi, Chem. Mater., 2011, 23, 285.

[3] A. Arakcheeva, D. Logvinovich, G. Chapuis, V. Morozov, S. V. Eliseeva, J.-C. G. Bunzli, P. Pattison, Chem. Sci., $2012,3,384$.

[4] J. Warczewski, Krystaliczne Struktury Modulowane, Third Edition, PWN, 1993.

[5] P. Coppens, V. Petricek, D. Levendis, F. K. Larsen, A. Paturle, Y. Gao, A. D. LeGrand, Phys. Rev. Lett., 1987, 59(15), 1965

[6] B. Pinheiro, A. M. Abakumov, IUCrJ, 2015, 2, 137.

[7] D. Tchoń, D. Trzybiński, A. Wrona-Piotrowicz, A. Makal, CrystEngComm, 2019, 21, 5845-5852

Keywords: incommensurately modulated structures, solid-state luminecence, 